\title{
The QoS-RWP Mobility and User Behavior Model for Public Area Wireless Networks
}

\author{
Giovanni Resta \\ IIT - CNR \\ Via G.Moruzzi 1, 56124 \\ Pisa - ITALY \\ giovanni.resta@iit.cnr.it
}

\author{
Paolo Santi \\ IIT - CNR \\ Via G.Moruzzi 1, 56124 \\ Pisa - ITALY \\ paolo.santi@iit.cnr.it
}

\begin{abstract}
Congestion is expected to become a prominent problem to deal with as the popularity of wireless data networks continues to increase. However, this problem can in principle be mitigated if a fraction of the network users could decide to move to another location in case their perceived QoS degrades. To account for this, we propose an extension of the well-known RWP model called QoS-RWP, in which users are divided into mobile users displaying constrained movement patterns, and QoS-driven users who are mainly stationary, but they can decide to move to a better location to improve their QoS level. Another enhancement of QoS-RWP with respect to the original RWP model is that waypoints are chosen according to an access point (AP) popularity metric, which reflects the recently observed phenomenon that different APs in a wireless data network display very different degrees of popularity among users. The QoS-RWP model also accounts for different classes of load offered to the network by the users, and for different channel access methods. Based on QoS-RWP, we perform a simulationbased analysis of network usage under different combinations of network parameters such as the number of users, number of APs, relative fraction of QoS-driven users, and channel access method. Our investigation discloses interesting insights on network usage, and shows that our model is able to capture important properties observed in real-world network deployments.
\end{abstract}

\section{Categories and Subject Descriptors}

C.2.1 [Network Arquitecture and Design]: Wireless communication

\section{General Terms}

Measurement, Performance

\section{Keywords}

Mobility modeling, Random Waypoint mobility, Quality of Service, Wireless LAN, Wireless Mesh Networks.

Permission to make digital or hard copies of all or part of this work for personal or classroom use is granted without fee provided that copies are not made or distributed for profit or commercial advantage and that copies bear this notice and the full citation on the first page. To copy otherwise, to republish, to post on servers or to redistribute to lists, requires prior specific permission and/or a fee.

MSWiM'06, October 2-6, 2006, Torremolinos, Malaga, Spain.

Copyright 2006 ACM 1-59593-477-4/06/0010 ...\$5.00.

\section{INTRODUCTION}

Thanks to the increasing popularity of 802.11-based products, wireless data networks have become widespread in recent years: medium- to large-scale WLAN deployments are nowadays common in campuses, enterprises, and so on, and also public areas such as airports, shopping malls, and parks, are becoming increasingly covered by wireless access points (APs). The proliferation of wireless data networks is expected to increase even further when the recently introduced wireless Mesh technology will become mature [7].

Given the proliferation of wireless devices, and the fact that data intensive multimedia applications such as video/audio streaming and on-line gaming are becoming increasingly popular, we can expect that network congestion will become a fundamental issue to solve in a near future. Actually, occasional network congestion (e.g., in a campus cafeteria at lunch time) has been observed in recent studies analyzing user traces collected in campus [16, 17], corporate [4] and conference [1] networks. However, we can expect that in a near future network congestion will become a relatively common situation, and strategies for minimizing the negative impact of network congestion on the user-perceived quality of service will be needed. Typical measures to address this need are network planning (e.g., deploying additional APs), and/or dynamic load balancing strategies, which both have pros and cons. It is widely acknowledged that both network planning and dynamic load balancing techniques could benefit a lot from the knowledge of typical user mobility and traffic patterns. This explains the great efforts that have been devoted in the literature to the characterization of user behavior in different environments [1, 4, 13, 16, 19, 20, 26, 27]. While these characterizations based on real-world traces can be very useful in the design of congestion relief techniques, they have the disadvantage of being tailored to a very specific scenario (e.g., the Dartmouth campus as in $[13,16]$ ), which in principle limits the usefulness of these characterizations to that specific scenario. Another disadvantage of using trace-based characterizations of user behavior is that they do not allow the designer to change many configuration parameters (e.g., number of users, number of APs, offered load, and so on), so that investigation of a certain congestion relief technique's performance in different network conditions is not possible.

The alternative to trace-based models of user behavior is to use synthetic mobility and traffic models. However, these models (especially for what concerns mobility) are often considered as too general, and not representative of any real-world scenario. Furthermore, popular mobility models 
such as the random waypoint model [15] have been recently shown to have properties such as non-uniform node spatial distribution [5] and speed decay on the long run [28], which could impair the accuracy of mobile network simulation.

In this paper, we extend the RWP model to account also for user behavior. The goal of our QoS-RWP model is to mimic a situation in which a fraction of the users displays constrained movement patterns (e.g., because they have to move to a certain specific location within a certain time), while the remaining users are mainly stationary, but they could decide to move to another location in case their perceived QoS degrades considerably. In the remainder of this paper, we call users of the former type mobile users, and users of the latter type $Q o S$-driven users. We believe distinguishing users into mobile and QoS-driven is representative of many WLAN or wireless Mesh deployments in public areas such as airports, parks, shopping malls, and so on, as acknowledged also in $[2,25]$. For instance, consider the case of a public park. In this situation, many users are seated somewhere in the park, using their wireless devices to browse the Web, sending emails, downloading music files to listen with the iPod, and so on. For this class of users, what is relevant is the perceived QoS: as long as the QoS is satisfactory, there is no reason to move to another location. However, if the QoS degrades below an acceptable level, a typical user of this class may decide to look for a better location in the park, becoming stationary again when the perceived QoS is restored to an adequate level. On the other hand, other users in the park scenario move intentionally in or across the network area, for instance because they are jogging while downloading and listening music in their MP-3 player, or because they are crossing the park in their way to the office while using a VoIP application. It is not difficult to imagine similar scenarios in other environments such as airports, shopping malls, campuses, and so on.

To model user traffic behavior, we classify users into a number of classes depending on the amount of offered load to the network. More specifically, we consider three classes of users, offering 'low' (e.g., light Web browsing, email, chat), 'medium' (e.g., intensive Web browsing, streaming audio, file download), and 'high' (e.g., streaming video, 3D on line gaming) load to the network, respectively. Note that this classification of users based on the offered load is (in principle) independent of their classification into mobile or QoSdriven.

In principle, the general idea of modeling QoS-driven user behavior is independent of the specific wireless technology used in the network. However, realistically modeling user perceived QoS level requires committing to a certain channel access technology, whose model is then also a fundamental building block of QoS-RWP. In this paper, we have modeled two possible channel access methods: data polling, which mimics the long-term behavior of $802.11 \mathrm{a} / \mathrm{b} / \mathrm{g}$ networks; and time polling, which mimics the long-term behavior of forthcoming 802.11e networks.

Another distinguishing feature of QoS-RWP is that waypoints of mobile nodes are not chosen uniformly at random in the deployment area as in the traditional RWP model, but according to an AP popularity metric. The fact that different APs in a wireless data network display very different popularity degrees (i.e., number of registered users) has been recently observed in different types of wireless data networks $[4,17,26]$. As such, we believe accounting for the popular- ity degree when choosing the next waypoint contributes to improving the accuracy of our model.

As an example of utilization of our mobility and user behavior model, we perform an extensive analysis of network usage under a wide range of different conditions in terms of number of users, number of deployed APs, percentage of QoS-driven users, and channel access method. The main findings of our analysis are that the presence of QoS-driven users can significantly increase the QoS-level in the network (especially when combined with the time polling channel access method), and that, in order to avoid resource wastage, network designers should carefully consider both the channel access method and the estimated fraction of QoS-driven users before deploying additional (possibly scarcely utilized) resources. Our analysis also shows that the QoS-RWP model is able to reproduce an important phenomenon which has been observed in real-world network deployments, i.e. the loose correlation between the number of registered users and the load observed at the APs.

Another example of possible utilization of our model is to generate user and traffic traces that can be used in the performance evaluation of wireless Mesh network protocols. Currently, the performance of these protocols is typically evaluated assuming that the number of registered users and/ or traffic load at each AP (wireless router, according to Mesh networks terminology) is generated randomly, typically using a uniform distribution between certain upper and lower bounds. As observed phenomena such as different AP popularity levels indicate, generating AP loads according to a purely random distribution could be very inaccurate, possibly leading to misleading conclusions about the performance of the investigated protocols. To improve accuracy when generating the user and traffic profiles, network designers can use the user and traffic traces generated by QoS-RWP to feed the custom simulator used to evaluate the performance of a specific protocol.

\section{RELATED WORK AND CONTRIBUTION}

The characterization of user behavior in wireless data networks has been subject of intensive research in recent years. By analyzing user traces collected at the various access points in campuses [16, 17], corporate buildings [4], or conferences [1], typical user behaviors have been analyzed, and phenomena such as different popularity levels of the APs, occasional network congestion, low correlation between the number of users and load, and so on, have been observed. More recently, some authors have derived synthetic models of user behavior which try to mimic the observed user behavior in a certain environment. This is the case, for instance, of the ModelT model proposed in [13], which is based on the traces collected from the Dartmouth College campus over a period of 2 years. This model has been recently extended to account for spatio-temporal correlation in the user registration patterns [20]. Other examples of synthetic mobility model based on real-world traces are proposed in [19, 27]. While allowing the network designer to have a certain degree of freedom in setting some network parameters such as number of users and access points, synthetic models based on real-world traces have the disadvantage of being representative of a specific scenario (that from which the traces were collected). Furthermore, these models are built to capture the characteristics of existing or past wireless networks, and 
it is not clear to what extent these models are representative of future wireless network deployments.

Another related active area of research in recent years is the analysis of existing 'general purpose' synthetic mobility models, such as the random waypoint and the random direction model, which, mostly due to their simplicity, are the most commonly used in the simulation of wireless ad hoc network protocols. Recent studies have characterized the long term node behavior in these types of mobile networks $[5,18,21,23,28,29]$, outlining some undesired phenomena such as node concentration in the center of the deployment region [5], and long term decay of the average nodal speed [28]. These phenomena could impair the accuracy of simulations based on these synthetic mobility models, essentially because the initial network conditions can be very different from the long term network conditions. Another acknowledged weakness of random waypoint/direction -like mobility is that it is too general, and not representative of any real-world scenario. In order to address some of the criticisms raised against random waypoint/direction mobility, some authors have recently proposed more realistic and/or more accurate synthetic mobility models, such as the obstacle model proposed in [14], and those proposed in [6, 12, 22, 29]. For a survey on mobility models for wireless ad hoc networks the reader is referred to [8].

Despite the considerable amount of research efforts referred above, to the best of our knowledge none of the existing synthetic models of user behavior in wireless networks is based on the fundamental observation that a fraction of users might not display constrained movement patterns, but they could decide to move to a better location in case their perceived QoS level drops below an acceptable level. We want to remark that the fact that some users might be willing to move to another location to improve their QoS has already been observed in [25], and explicitly suggested as a method to alleviate congestion in [2]. In the latter paper, the authors introduce the concept of network-directed roaming, which can be briefly explained as follows: if the required QoS cannot be guaranteed to a certain user, the network itself indicates to the user where to roam in order to obtain the desired level of service. This technique, combined with explicit channel switching, is shown to considerably improve load balancing in the network.

The main contribution of this paper is the proposal of a mobility and user behavior model that explicitly takes the degree of user satisfaction into account. The need of estimating the degree of user satisfaction complicates a lot the model definition, since aspects such as user offered load and channel access method have to be carefully modeled. To keep the complexity of our proposed model to a reasonable level, we have decided to simplify the mobility part as much as possible, while at the same time accounting for relevant aspects such as degree of AP popularity. This explains our choice of modeling user movements according to the well-known RWP model, suitably modified to choose the waypoints according to an AP popularity metric.

As an example of QoS-RWP utilization, we also present an extensive analysis of network usage under different scenarios. Note that, differently from [2], in this paper we are not proposing any explicit congestion relief technique. Instead, motivated by previous work showing the beneficial effect of QoS-driven users on load balancing [2], our aim is to carefully investigate the effects on network congestion of having a fraction of QoS-driven users. This outlines another major difference with respect to [2], in which it is implicitly assumed that all the users are QoS-driven. We believe that in most real world scenarios only a fraction of the users can be classified as QoS-driven, while other users display a constrained movement pattern. Another difference with respect to [2] is that we are not assuming network-directed roaming, in which a users decide to move after an explicit roaming message sent by the network manager. Instead, in our model we assume that it is the user himself, based on the perceived QoS level, who decides when to start moving, and the destination of the movement.

In order to achieve our goal of investigating the effect of QoS-driven mobility on network congestion, we consider a wide range of parameter settings, including not only the relative fraction of QoS-driven users, but also the number of users, the number of APs, the channel access method, and so on. To the best of our knowledge, this is the first such study presented in the literature.

\section{QOS-RWP}

In this Section we introduce our mobility and user behavior model, which we call QoS-RWP. QoS-RWP is composed of three main components: mobility modeling, traffic modeling, and channel access modeling. We describe each of these components in a separate subsection.

\subsection{Mobility model}

As mentioned in the previous sections, in QoS-RWP we assume that a certain fraction $f$, with $0 \leq f \leq 1$, of the $n$ network users is QoS-driven, while the remaining $(1-f) \cdot n$ users display constrained movement patterns.

Movement of QoS-driven users obeys the following rules. Users in this class are mostly stationary, i.e. they are not willing to move unless their perceived QoS level drops below an acceptable threshold. To determine whether the QoS level is acceptable for user $u$, we measure the number of buffer overflow/underflow (depending on whether we are sending or receiving packets) experienced by $u$ during the last $\Gamma$ seconds, where $\Gamma$ is a tunable parameter which can be used to smooth temporary variations in the QoS level. The status of the buffer is checked every $\gamma$ seconds, with $\gamma \ll \Gamma$, where a check is successful if there is no overflow/underflow, unsuccessful otherwise. If at least a fraction of $r q(u)$ checks are successful, where $r q(u)$ is a tunable parameter modelling the minimum QoS level that user $u$ considers acceptable, then $u$ is satisfied, otherwise it is unsatisfied and decides to change AP association and/or to move.

Since we assume that QoS-driven users are 'lazy', and that the only reason forcing them to move is low QoS level, in our model we assume that a user first tries to do a 'virtual movement' by simply changing AP association. Using the terminology introduced in [2], we call this action channel switching.

In case channel switching is possible, user $u$ changes AP association, selecting the AP which provides him/her the best QoS. Estimating the QoS level achieved by surrounding APs is not immediate, since the user perceived QoS depends not only on the quality of the wireless channel connecting to the AP, but also on the load offered by the other users registered at that AP. Hence, estimating the QoS achieved by surrounding access points requires information exchange between the user and the APs, which might not be allowed 
or implemented in certain network deployments. For this reason, in QoS-RWP we simply assume that the user selects the AP to switch to based on the signal quality, preferring the AP with the best signal (and, consequently, data rate).

Note that in QoS-RWP we are assuming that a typical network user is essentially selfish, i.e., he/she tries to improve his/her own QoS level, without considering possible degradation of the QoS level of other users. An alternative is to assume that users are naturally willing to cooperate with each other in order to optimize network performance, as it is assumed, for instance, in [2]. However, cooperative user behavior is a reasonable assumption only in networks composed of 'homogeneous' users, such as corporate networks. On the other hand, networks deployed in public areas are likely to be used by very 'heterogeneous' users, and assuming spontaneous cooperation between users seems a bit optimistic. This explains our choice of assuming selfish user behavior.

In case channel switching is not possible (because there is no overlapping of APs in the current user's position, or because also the other APs are overloaded), an unsatisfied QoS-driven user decides to move. Again, there are many different options to model movement of an unsatisfied user. To keep the model simple, we assume that unsatisfied QoSdriven users move according to the same rules governing the movement of mobile users, i.e. AP popularity weighted RWP movement (see below).

Let us now consider mobile users. In order to reduce the complexity of our model, we assume that mobile users follow a weighted waypoint mobility model similar to that proposed in $[12]^{1}$. Mobile users alternate between pause times at waypoints and traveling periods between successive waypoints. Initially, a user is assigned a certain waypoint according to a weighted, non uniform distribution which resembles the different popularity of APs. The fact that APs in a wireless data network display different popularity degrees is well documented in the literature $[1,4,17]$. In our model, we initially assign each AP $a$ with a popularity degree $p(a)$ according to a probability distribution resembling a power law (as suggested in [13]). When selecting the initial or a new waypoint for a certain mobile user, first an AP is selected with a probability proportional to its popularity, then a waypoint is selected uniformly at random in a bounded vicinity of the selected AP. The pause time at waypoints is chosen according to a Poisson distribution with intensity $\lambda_{p}$. In order to avoid degenerate situations of excessively long or short pause times, we have imposed an upper and lower bound to the pause time at the waypoints. The trajectory between consecutive waypoints is linear, and the velocity is chosen uniformly at random in an interval $\left[v_{\min }, v_{\max }\right]$. Since in this paper we are primarily concerned with pedestrian users, we set $v_{\min }=v_{\max }=1 \mathrm{~m} / \mathrm{sec}$.

\subsection{Traffic model}

In QoS-RWP, users are divided into three different classes of offered load: low, medium, and high load. The lowest

\footnotetext{
${ }^{1}$ We are aware that more realistic mobility models such as, for instance, the obstacle model of [14], could have been used in QoS-RWP. However, this would have increased a lot the complexity of the simulator and the simulation running time. For this reason, we have opted for a simple model such as random waypoint mobility, but enriched with 'more realistic' salient features such as weighted choice of waypoints according to AP popularity.
}

class of traffic accounts for users who are using the network for emailing, chatting, and light web browsing. The average bandwidth requirement of this class of users is a tunable parameter, but the actual bandwidth requirement is very variable, since users in this class typically generate bursty traffic. In the study reported in sections 4 and 5, the average bandwidth requirement of this class of users is set to 64 $\mathrm{Kb} / \mathrm{sec}$.

The medium class of traffic accounts for users who are using the network for intensive web browsing, file downloading, audio streaming, and so on. The average user bandwidth requirement in this case is also a tunable parameter, and the generated traffic displays a more uniform pattern. To maintain the complexity of the simulator at a reasonable level, we assume users in this class generate UDP-like traffic. In the study reported in the following, we have set the average bandwidth requirement of medium users to $256 \mathrm{~Kb} / \mathrm{sec}$.

Finally, the highest class of traffic accounts for users who make an intensive use of the network, such as video streaming, 3D online gaming, and so on. The average user bandwidth requirement is again a tunable parameter. Similarly to the case of medium traffic, also high traffic users typically display a relatively uniform bandwidth usage. Hence, we assume UDP-like traffic for these users, with a rate which is set to $2 \mathrm{Mb} / \mathrm{sec}$ in the study reported in the following.

The allocation of users to the various traffic classes is performed according to a certain mix $l, m, h$, where $l$ is the fraction of low traffic users, $m$ is the fraction of medium traffic users, and $h=1-(l+m)$ is the fraction of heavy traffic users.

\subsection{Channel model}

In QoS-RWP, we model the radio channel as follows. For given user $u$ and $\mathrm{AP} a$, we estimate the quality of signal between $u$ and $a$ according to a radio propagation model. In the current version of the simulator, we have considered free-space and log-normal shadowing propagation, but other propagation models can easily be integrated into QoS-RWP. The quality of signal is used to determine the achievable data rate between $u$ and $a$, if $u$ is actually within the coverage range of AP $a$. We have considered the eight possible data rates available in 802.11 a, i.e. $6,9,12,18,24,36,48$ and $54 \mathrm{Mbs}$. The nominal radio range of an AP at the various data rates is set according to what reported in the data sheets of the CISCO Aironet 1240AG access point [9]. The nominal ranges are used in combination with the free space radio propagation model to determine the highest possible available data rate between $u$ and $a$. In case of log-normal shadowing, the distance between $u$ and $a$ cannot be directly converted into a data rate value, due to the random component in the signal attenuation due to shadowing. Hence, the geographical distance between $u$ and $a$ is converted into a 'virtual distance' accounting for the actual signal attenuation between $u$ and $a$, and the highest available data rate is obtained by comparing this 'virtual distance' with the nominal radio ranges.

As mentioned in the Introduction, QoS-RWP implements two channel access methods: data polling and time polling. Data polling is similar to the $802.11 \mathrm{a} / \mathrm{b} / \mathrm{g}$ MAC layer with PCF coordination: registered users are polled by the AP. In case a polled user $u$ has a packet to send/receive to/from the $\operatorname{AP} a$, the packet is sent at the highest possible data rate available between $u$ and $a$. If a polled user has nothing 
to send/receive, the next registered user is polled, and so on. Note that, from a high level point of view, there is little difference between DCF and PCF coordination in 802.11, since DCF is designed to give the same long term probability of accessing the channel to contenders [11]. Hence, our data polling model is representative also of $802.11 \mathrm{a} / \mathrm{b} / \mathrm{g}$ networks with DCF coordination.

In order to keep the complexity of simulation at a reasonable level, in QoS-RWP we have not implemented any exchange of control messages. However, we have accounted for the overhead caused by control message exchange and channel access time by using an actual data rate for sending packets which is significantly lower than the nominal data rate. In particular, we have used the actual data rates reported in Table 1, which are obtained from the measurements reported in [10].

Table 1: Actual data rates (in Mbs) achieved with 802.11a.

\begin{tabular}{|c|c|c|c|}
\hline Nominal & Actual & Nominal & Actual \\
\hline \hline 54 & 25 & 18 & 12.8 \\
\hline 48 & 24 & 12 & 9.6 \\
\hline 36 & 19.5 & 9 & 7.2 \\
\hline 24 & 16 & 6 & 5.4 \\
\hline
\end{tabular}

The current implementation of the $802.11 \mathrm{a} / \mathrm{b} / \mathrm{g}$ MAC layer is known to have major inefficiencies. In particular, both DCF and PCF access methods suffer when registered users have different data rates, because the slowest user reduces the throughput of the other users down to the value of the slowest user ${ }^{2}$. This performance anomaly, which has been observed in [11], is caused by the data polling approach, in which a user, once gained access to the channel, transmits a packet, independently of his/her data rate. Hence, the time allotted to each user can be very different, and slower users tend to use the channel for longer times, driving down the throughput of faster users.

The second access model used in QoS-RWP, time polling, solves this problem by assigning to each polled user a time slot, instead of whatever time is necessary to send a packet. With this approach, all the users gain access to the channel for an approximately equal share of time, and faster users can transmit more packets than slower ones. Time polling resembles the basic functioning of the 802.11e MAC extension for QoS support, which is in the final stage of standardization.

A final consideration about channel access in QoS-RWP is that we assume adjacent APs use different (orthogonal) channels, so that interference between users registered at adjacent APs is not an issue.

\section{ANALYSIS OF NETWORK USAGE}

In the following sections, we report an extensive study of network usage under different scenarios performed using our proposed QoS-RWP model. We start by presenting the simulation setting. The results of our study are reported in the next section.

${ }^{2}$ This is true under the assumption that all the registered users always have a packet to send in their queues.

\subsection{AP and user distribution}

A number $m$ of APs is distributed in a square deployment region $R$, which is assumed to have a $1 \mathrm{Km}$ long side. APs are distributed according to a 'guided uniform' distribution, which is designed to provide at least a certain degree of coverage of the deployment region. More specifically, $R$ is divided into 25 squares of side $200 \mathrm{~m}$, and approximately $\mathrm{m} / 25$ APs are distributed uniformly at random in each square. If $m$ is below $25, m$ squares are randomly selected in the deployment region, and one AP is distributed uniformly at random in each of the selected squares. Each deployed AP $a$ is then assigned with a degree of popularity $p(a)$, which reflects its potential of attracting network users. The degree of popularity of APs is computed according to a probability distribution resembling a power law (see [13]).

After AP deployment, a number $n$ of users is distributed in the network according to the following rules. First, each user is assigned a mobility class (QoS-driven/mobile) and a traffic class (low/medium/high) according to the defined parameters $f, l, m$, and $h$. Then, he/she is assigned with an initial position by first randomly selecting an AP according to its popularity, and then choosing a position uniformly at random in a bounded vicinity (within the coverage area) of the selected AP. To model users joining/leaving the network, we assume that each user is randomly assigned an active/sleep state, where the active/sleep transition is governed by a Poisson law with parameter $\lambda_{a}$, and the sleep/active transition is governed by a Poisson law with parameter $\lambda_{s}$. Hence, $n$ must be intended as the maximum possible number of network users, and the actual number of users using the network at a given time is in general lower. In order to avoid degenerate situations of excessively long or short transition times, we have imposed an upper and lower bound to the active/sleep and sleep/active transition time.

In all the experiments reported in the following, the simulated network time is 14400 seconds (4 hours).

\subsection{Performance metrics}

To characterize network usage under different conditions, we compute the following metrics as a result of our simulations:

total network load (NL): total number of packets exchanged in the network during the simulated time interval. packet delivery rate: we trace the average packet delivery rate experienced by network users during the last 60 seconds; we also compute the average packet delivery rate experienced by network users during the entire simulated time interval.

balance index: let $L_{i}$ denote the load observed at the $i$-th AP. The balance index [2] is defined as

$$
\beta=\frac{\left(\sum_{i=1}^{m} L_{i}\right)^{2}}{m \cdot \sum_{i=1}^{m} L_{i}^{2}} .
$$

The balance index is used to measure the deviation in utilization of the different APs: if the load is equally divided amongst the APs, we have $\beta=1$; conversely, with highly unbalanced network load we have $\beta \approx 1 / \mathrm{m}$. Similarly to the packet delivery rate, the balancing index is averaged both in 60 seconds intervals, and in the entire simulation time. prevalence and persistence: these metrics, which are used also in [4], model the mobility of users independently of the duration of simulation and of the amount of time a user spends in the network. Prevalence of user $u$, denoted 
Table 2: Parameters used in the QoS-RWP model and in our simulations.

\begin{tabular}{|c|c|c|}
\hline Parameter & Meaning & Values \\
\hline$m$ & number of APs & $25,30,40,50$ \\
\hline & simulated time interval & $14400 \mathrm{sec}$ ( 4 hours $)$ \\
\hline$p(a)$ & degree of popularity of AP $a$ & chosen according to a power law \\
\hline$n$ & maximum number of users & $100-800$ \\
\hline$\lambda_{a}$ & intensity of Poisson law governing active/sleep transition & 5 min. (min) - 90 min. (avg) - $180 \min ,(\max )$ \\
\hline$\lambda_{s}$ & intensity of Poisson law governing sleep/active transition & 5 min. (min) - 20 min. (avg.) - 90 min. ( $\max )$ \\
\hline$r q(u)$ & required QoS value for user $u$ & 0.90 \\
\hline$\Gamma$ & window lenght to determine user satisfaction at current location & 5 sec. \\
\hline$\gamma$ & buffer check interval & $0.05 \mathrm{sec}$ \\
\hline$\lambda_{p}$ & intensity of Poisson law governing pause times at waypoints & 5 min. (min) - 60 min. (avg) - $120 \min .(\max )$ \\
\hline$v$ & velocity of mobile users & $1 \mathrm{~m} / \mathrm{sec}$ \\
\hline$f$ & fraction of QoS-driven users & $0,0.125,0.25,0.5$ \\
\hline$l, m, h$ & relative fraction of low, medium, and high traffic users & $0.33,0.33,0.33$ \\
\hline pathLoss & radio propagation model & free space \\
\hline packSize & packet size (in the data polling model) & $1 K b$ \\
\hline timeSlot & time slot length (in the time polling model) & $200 \mu \mathrm{sec}$ \\
\hline$N L$ & total network load & \\
\hline$P D R$ & packet delivery ratio & \\
\hline$\beta$ & balance index & \\
\hline $\operatorname{prev}_{q}(u)$ & prevalence of QoS-driven user $u$ & \\
\hline $\operatorname{pers}_{q}(u)$ & persistence of QoS-driven user $u$ & \\
\hline $\operatorname{prev}_{m}(u)$ & prevalence of mobile user $u$ & \\
\hline $\operatorname{pers}_{m}(u)$ & persistence of mobile user $u$ & \\
\hline
\end{tabular}

$\operatorname{prev}(u)$, measures the overall fraction of time user $u$ spends with the AP is registered with for a longer time. Prevalence accounts for the total registration time with a certain $\mathrm{AP}$, and does not take into account the duration of each session with the given AP. To account for this, we consider also persistence, denoted pers $(u)$, which measures the average amount of time user $u$ stays associated with an access point before being forced to move to another AP or leaving the network. We separately compute these metrics for QoS-driven and mobile users.

The many parameters used in QoS-RWP and in our simulations are summarized in Table 2 .

\section{SIMULATION RESULTS}

\subsection{Increasing number of users}

In the first set of experiments, we have analyzed network usage as the number of APs is fixed to 25, and the maximum number of users increases from 100 to 800 . We have considered both channel access methods, and different fractions of QoS-driven users. The other simulation parameters are summarized in Table 2. The purpose of these experiments is to investigate how network usage changes as the network becomes more and more saturated (the number of AP is fixed, and the number of users increases). Due to the relatively long simulation time ${ }^{3}$ and the many different parameter settings used in our experiments, we have used a sample size of 3 simulation runs ${ }^{4}$. For the same reason, we have used only the free space propagation model in our experiments.

Figure 1 reports the average packet delivery rate at the end of the 14400 seconds of simulated time for increasing

${ }^{3}$ Depending on the channel access method (time polling is about twice as fast as data polling) and the machine, the simulation time of a single instance with 800 users varied between $24 \mathrm{~min}$. and $90 \mathrm{~min}$.

${ }^{4}$ We have verified in preliminary simulation runs that the variance is already acceptable with a sample size of 3 runs. values of $n$ and different values of $f$. As seen from the graphics, QoS-driven users have a beneficial effect on the PDR: as $f$ varies from 0 to 0.5 , the PDR value increases from 0.66 to 0.75 with data polling channel access and 800 users ( $14 \%$ increase), and from 0.68 to 0.82 in case of time polling ( $19 \%$ increase). Hence, time polling channel access has the potential of getting more benefits than data polling from having a fraction of QoS-driven users in the network. Concerning the relative performance of time polling vs. data polling access method, using time polling increases PDR of about $4 \%$ with respect to data polling with 800 users when $f=0$, and of about $9 \%$ when $f=0.5$.

Similar trends can be observed for the total network load, which is reported in Figure 2. In this case, the performance increase of having a fraction 0.5 of QoS-driven users is $12 \%$ in case of data polling, and $18 \%$ in case of time polling (when $n=800$ ). Concerning the relative advantage of time polling over the data polling channel access method, it is about $4 \%$ when $f=0$, and about $9 \%$ when $f=0.5$.

The balance index at the end of the simulated time interval for increasing values of $n$ and different values of $f$ is reported in Figure 3. As seen from the plots, the major factor influencing $\beta$ is the number of users $n$, while the channel access method and the fraction of QoS-driven users seem to have little influence on $\beta$. The balance index shows a decreasing trend until $n$ is $200 / 300$, then it starts to increase with $n$. This behavior is displayed independently of the channel access method and of the fraction of QoS-driven users. A possible explanation for this behavior is the following. When $n$ is relatively low $(n \leq 200)$, the network is far from saturation, and QoS-driven users tend to remain stationary. Given the relatively low number of active users (when $n=100$, even if all the users are awake an average of only 4 users is registered per access point), the different popularity degree of the APs does not manifest itself to a great extent (essentially, because the sample size is very small). As the number of users increases and the network is still far from the saturation point $(n=200 / 300)$, the 

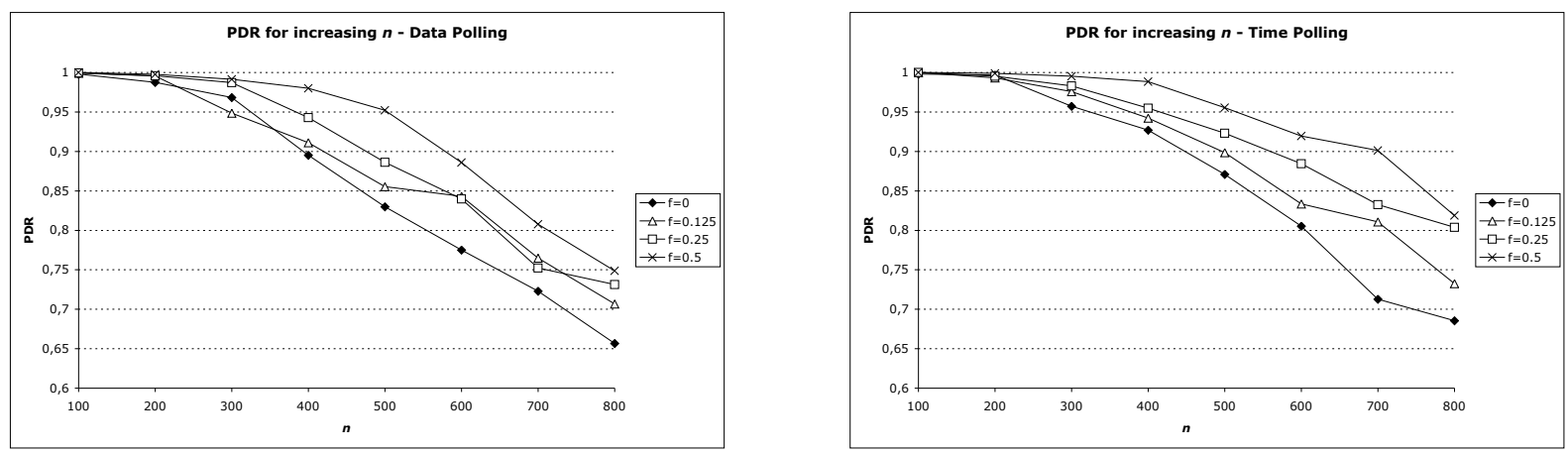

Figure 1: Packet Deliver Rate for increasing values of $n$ and different values of $f$. Channel access method is Data Polling (left) and Time polling (right).
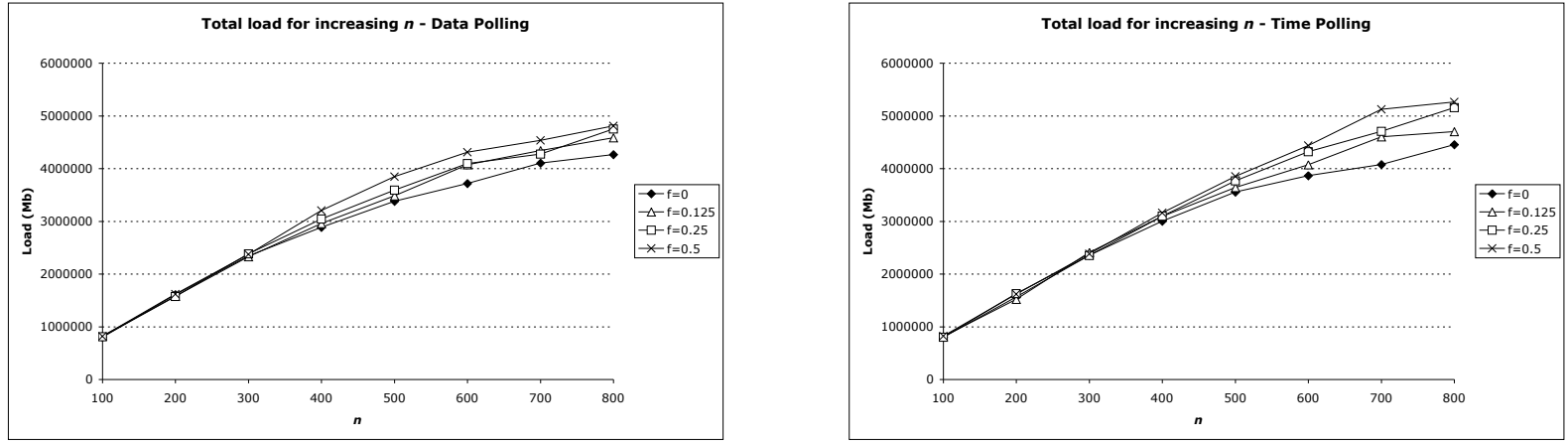

Figure 2: Total network load for increasing values of $n$ and different values of $f$. Channel access method is Data Polling (left) and Time polling (right).

different popularity degree of the APs manifests itself to a greater extent, and load unbalancing between the various APs emerges. However, as the number of users increases even more $(n \geq 400)$, the network becomes saturated, and an increasing number of unsatisfied QoS-driven users move across the network, improving load balancing.

Although referring to a quite different scenario, it is worth qualitatively discussing the results concerning the balance index obtained in our simulations and those reported in [2]. The simulation setting of [2] is the following: 100 users, 6 APs, data polling channel access scheme. The authors show that the balance index is increased of about $30 \%$ when the fraction of QoS-driven users increases from 0 to 1 . We recall that in [2] unsatisfied QoS-driven users are explicitly directed to another area of the network, so that network capacity is maximized (directed roaming). In our experiments, the fraction of QoS-driven users is at most 0.5 and, most importantly, QoS-driven users are not explicitly directed to 'optimal' network locations, but they instead move 'at random' (if channel switching is not possible), according to the popularity degree based RWP model described in Section
3.1. Nevertheless, when $n=100$ and channel access method is data polling, increasing the fraction of QoS-driven users from 0 to 0.5 results in a $22 \%$ improvement of the balance index, which is comparable to the improvement reported in [2]. This seems to indicate that somewhat random movement of QoS-driven users is in principle sufficient to achieve significant load balance increase.

Figure 4 shows the prevalence and persistence of QoSdriven and mobile users for increasing values of $n$ when the fraction of QoS-driven users is $f=0.5$. Both prevalence and persistence of mobile users are not influenced by $n$, nor by the channel access method. This is reasonable, since the movement pattern of mobile users is not influenced by the perceived QoS-level, nor by the number of active users in the network. The situation is different for QoS-driven users, who decide to change AP association and/or to move when the perceived QoS level is not satisfactory. Hence, we can expect that both prevalence and persistence are proportional to the fraction of satisfied QoS-driven users in the network. This behavior can actually be observed in Figure 4: as $n$ increases, the network load gets closer to the satura- 

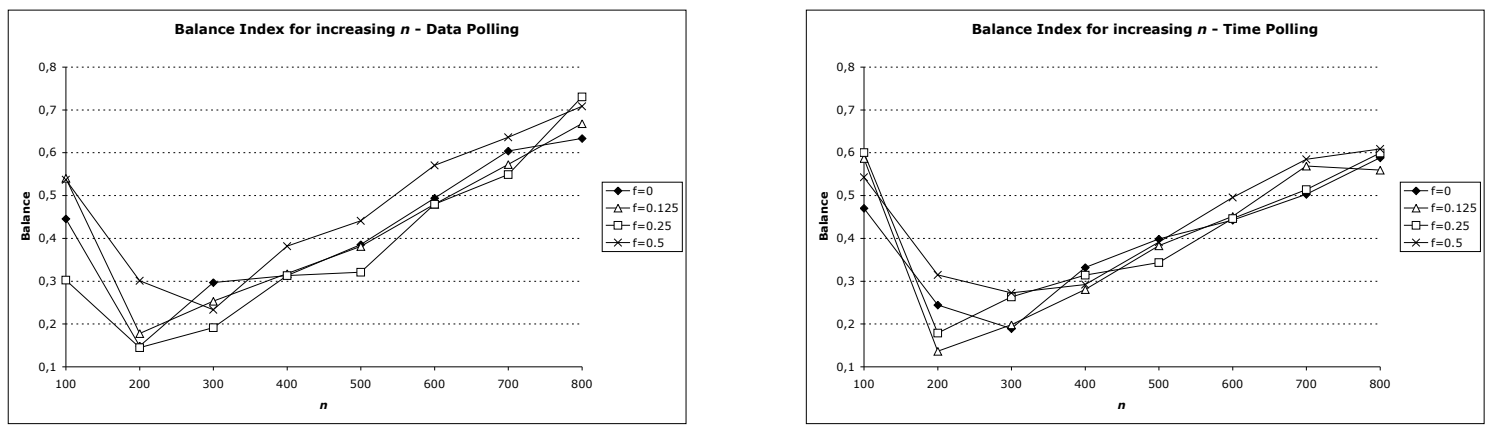

Figure 3: Balance index for increasing values of $n$ and different values of $f$. Channel access method is Data Polling (left) and Time polling (right).
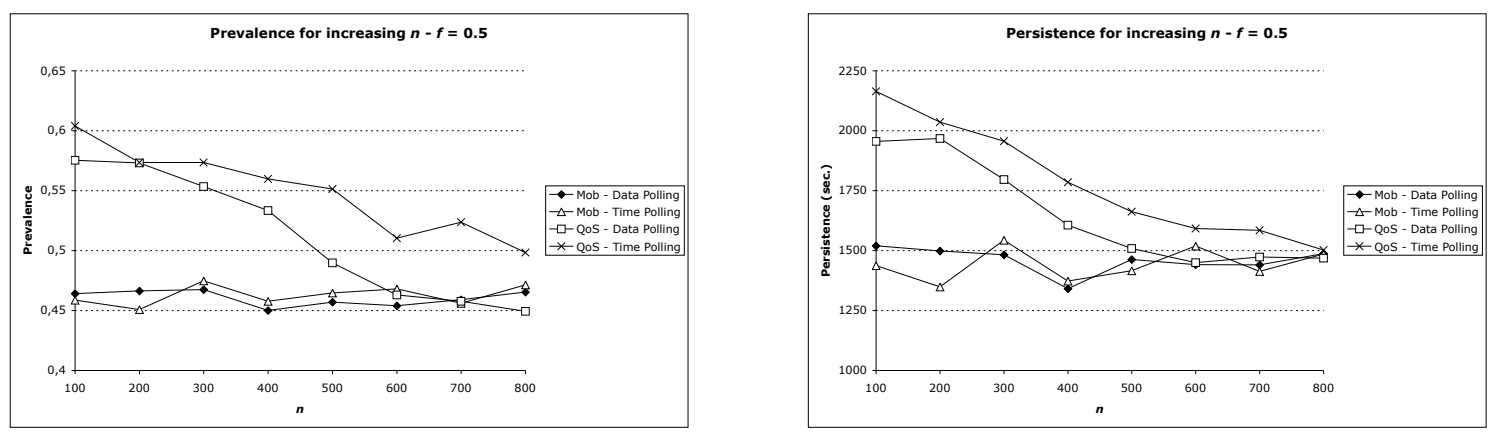

Figure 4: Prevalence (left) and persistence (right) for increasing values of $n$ with $f=0.5$.

tion point, and the fraction of unsatisfied QoS-driven users increases. As a consequence, both prevalence and persistence of QoS-driven users decrease with $n$. Comparing data polling with time polling channel access method, we observe a relatively higher prevalence and persistence with the time polling method, which reflects the fact that the average QoS level in the network is higher with time polling with respect to the case of data polling (see Figure 1).

In order to get a better understanding of the network usage evolution with time, we report the traces obtained from a single simulation run, referring to the following scenario: $n=800, f=0.5$, time polling channel access method. Figure 5-left reports the load vs. time registered at the maximally loaded ( $\max$ AP), minimally loaded (min AP), and 'close to average' AP (avg AP) at the beginning of the simulated time interval. The 'close to average' AP is chosen by first computing the average AP load at the beginning of the simulated time interval, and then selecting the AP whose load is closer (in absolute value) to the computed average. Figure 5-right reports the number of registered users vs. time for the same three APs. It is interesting to note that the load at the max AP changes a lot during the simulated time interval, and shows a decreasing trend. We believe this relatively unstable load observed at the max AP is due to the presence of QoS-driven users: when the load is very high, users becomes unsatisfied, and if a few of them are QoS-driven they decide to change AP association, causing a sudden decrease of the max AP load. However, the max AP is likely to have a relatively high popularity degree, attracting a relatively high number of mobile and/or newly activated users. Thus, the load at the max AP tends to increase again, until saturation is reached again, and so on. On the other hand, the observed load at the avg AP is more stable, and shows a slightly increasing trend. Combining the fact that the load observed at the max AP shows a decreasing trend, and the load at the avg AP shows an increasing trend, we can conclude that load balancing is improved with respect to the initial network conditions. As mentioned above, this better load balancing at the end of the simulated time interval is due to the presence of QoSdriven users.

Another interesting observation is the relatively low correlation between the observed load at the AP (Figure 5-left) and the number of registered users at the same AP (Figure 5-right): while the number of registered users at the avg AP is consistently higher than that observed at the $\max \mathrm{AP}^{5}$

${ }^{5}$ We recall that max and avg refers to the load, not to the number of users. 

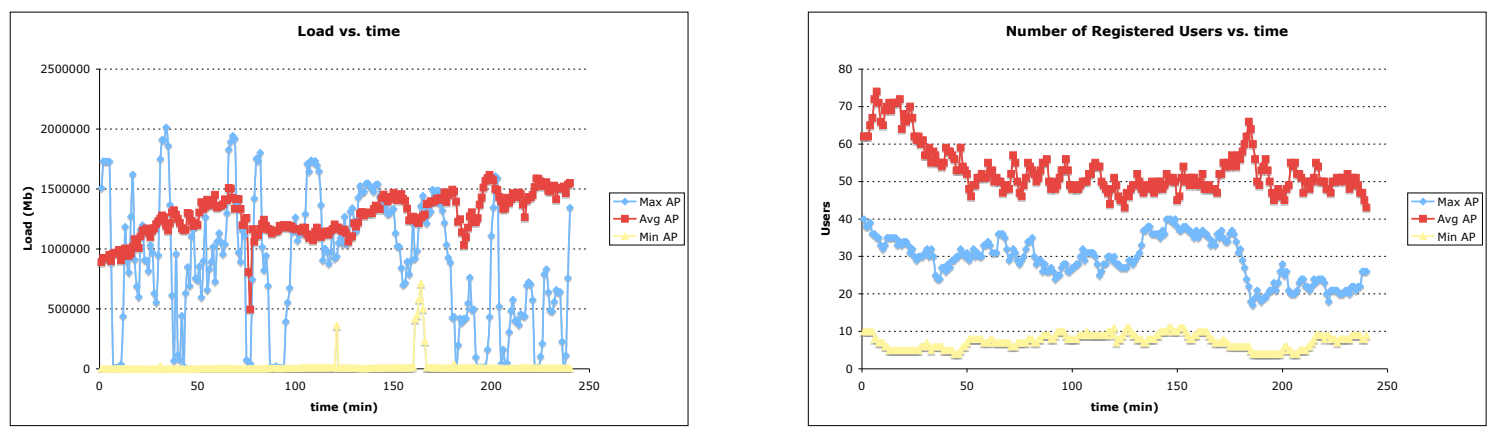

Figure 5: Total load (left) and number of registered users (right) at the maximally loaded, minimally loaded, and 'close to average' loaded access point, as a function of the simulated time. Parameter $n$ is set to 800, $f$ is 0.5 , and channel access method is time polling.

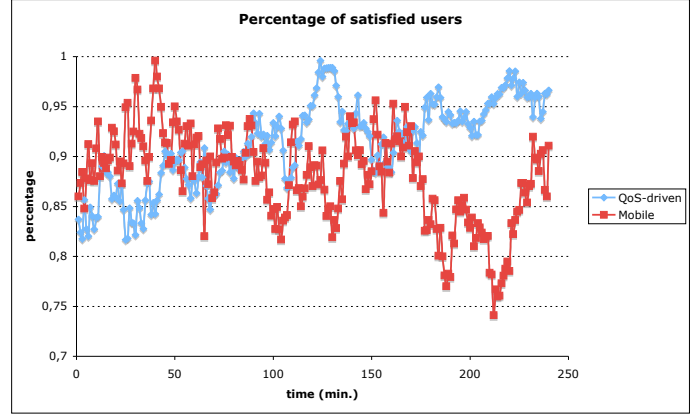

Figure 6: Fraction of QoS-driven and mobile satisfied users, as a function of the simulated time. Parameter $n$ is set to $800, f$ is $\mathbf{0 . 5}$, and channel access method is time polling.

(62 vs. 40 at the beginning of the simulated time interval), the observed load at the max AP is often considerably higher than that observed at the avg AP. We want to remark that the fact that the load observed at the APs and number of registered users are only loosely correlated have been observed in several papers that have analyzed user traces collected from real world network deployments $[1,4,17$, 26]. Thus, our QoS-RWP model is able to reproduce one of the most important behaviors observed in real-world deployments of wireless data networks, i.e. the low correlation between the number of registered users at an AP and the observed load at the AP.

Figure 6 reports the fraction of satisfied QoS-driven users over the total number of active QoS-driven users vs. time, and the same fraction vs. time relative to mobile users ${ }^{6}$, for the same simulation run as above. It is interesting to note that, while at the beginning of the simulated time interval

${ }^{6}$ Our simulator computes the perceived QoS level also for mobile users, although it has no influence on their behavior.
QoS-driven users have approximately the same degree of satisfaction of mobile users, as time goes by QoS-driven users increases their degree of satisfaction, which becomes considerably higher than that observed by mobile users. This is a consequence of the fact that, contrary to mobile users, QoS-driven users change their behavior depending on the experienced QoS-level.

\subsection{Increasing AP density}

Due to space limitations, the results of this set of simulations are reported in the full version of the paper [24].

\section{CONCLUSIONS}

In this paper, we have introduced the first synthetic mobility and user behavior model which explicitly takes the degree of user satisfaction into account. In particular, in our QoS-RWP model a fraction $f$ of the users is assumed to change AP association and/or to move in case the perceived QoS-level drops below an acceptable level. QoS-RWP is composed of three main components: mobility model, traffic model, and channel access model.

Based on QoS-RWP, we have performed an investigation of network usage under different conditions, which has disclosed interesting insights, and has shown that our model is able to reproduce phenomena which have been observed in real-world deployments (different AP popularity degrees, low correlation between number of registered users and load observed at the APs). As such, we believe our model can be successfully used in the simulation of wireless data networks, such as public area WLAN and wireless Mesh deployments. In this respect, our model can be considered as a response to the need of more realistic, yet simple, mobility and user behavior models for wireless hotspots outlined in [3]. With respect to recent models such as those proposed in $[13,19$, 20,27 , our model is more general and flexible, as several parameters such as number of users and APs, traffic mix, fraction of QoS-driven users, and channel access method can be easily modified.

We plan to prepare an open source, public release of our QoS-RWP model, so that it can be used and extended by other researchers in the field. We also plan to use the traces generated by our model to accurately investigate the perfor- 
mance of different WLAN and wireless Mesh network protocols.

\section{ACKNOWLEDGEMENTS}

The authors wish to thank Mauro Leoncini for actively participating in many discussions in the early stage of QoSRWP definition, and Doug Blough for reading a first draft of this paper, and giving several useful suggestions for improving our model.

\section{REFERENCES}

[1] A. Balachandran, G.M. Voelker, P. Bahl, P. Venkat Rangan, "Characterizing User Behavior and Network Performance in a Public Wireless LAN", Proc. ACM Sigmetrics 02, pp. 195-205, 2002.

[2] A. Balachandran, P. Bahl, G.M. Voelker, "Hot-Spot Congestion Relief and User Service Guarantees in Public-Area Wireless Networks", Proc. IEEE Workshop on Mobile Computing System and Applications (WMCSA), 2002.

[3] A. Balachandran, G.M. Voelker, P. Bahl, "Wireless Hotspots: Current Challanges and Future Directions", Mobile Networks and Applications, Vol. 10, pp. 265-274, 2005.

[4] M. Balazinska, P. Castro, "Characterizing Mobility and Network Usage in a Corporate Wireless Local-Area Network", Proc. ACM MobiSys 03, pp. 303-316, 2003.

[5] C. Bettstetter, G. Resta, P. Santi, "The Node Distribution of the Random Waypoint Mobility Model for Wireless Ad Hoc Networks", IEEE Trans. on Mobile Computing, Vol. 2, n.3, pp. 257-269, July-Sept. 2003.

[6] C. Bettstetter, "Smooth is Better than Sharp: A Random Mobility Model for Simulation of Wireless Networks", in Proc. ACM Intern. Workshop on Modeling, Analysis, and Simulation of Wireless and Mobile Systems (MSWiM), (Rome, Italy), pp. 19-27, July 2001.

[7] R. Bruno, M. Conti, E. Gregori, "Mesh Networks: Commodity Multihop Ad Hoc Networks", IEEE Communications Magazine, Vol. 43, n. 3, pp. 123-131, March 2005.

[8] T. Camp, J. Boleng, V. Davies, "Mobility models for ad hoc network simulations", Wireless Communication \& Mobile Computing (WCMC), special issue on mobile ad hoc networking, Wiley, 2002.

[9] Cisco Aironet 1240AG data sheets, available at http://www.cisco.com/en/US/products/hw/ /wireless.

[10] I. Haratcherev, J. Taal, K. Langendoen, R. Legendijk, H. Sips, "Automatic IEEE 802.11 Rate Control for Streaming Applications", Wireless Communications and Mobile Computing, Vol. 5, pp. 421-437, 2005.

[11] M. Heusse, F. Rousseau, G. Berger-Sabbatel, A. Duda, "Performance Anomaly of 802.11b", Proc. IEEE Infocom 03, 2003.

[12] W. Hsu, K. Merchant, H. Shu, C. Hsu, A. Helmy, "Preference-based Mobility Model and the Case for Congestion Relief in WLANs using Ad Hoc Networks", Proc. IEEE VTC 04, 2004.
[13] R. Jain, D. Lelescu, M. Balakrishnan, "Model T: and Empirical Model for User Registration Patterns in a Campus Wireless LAN", Proc. ACM Mobicom 05, pp. 170-184, 2005.

[14] A. Jardos, E. M. Belding-Royer, K. Almeroth, S. Suri, "Towards Realistic Mobility Models for Mobile Ad Hoc Networks", Proc. ACM MobiCom, pp. 217-229, 2003.

[15] D.B. Johnson, D.A. Maltz, "Dynamic Source Routing in Ad Hoc Wireless Networks", Mobile Computing, Kluwer Academic Publishers, pp. 153-181, 1996.

[16] M. Kim, D. Kotz, "Modeling Users' Mobility among WiFi Access Points", Proc. WiTMeMo 05, 2005.

[17] D. Kotz, K. Essien, "Characterizing Usage of a Campus-Wide Wireless Network", Proc. ACM Mobicom, pp. 107-118, 2002.

[18] J.Y. LeBoudec, M. Vojnovic, "Perfect Simulation and Stationarity of a Class of Mobility Models", Proc. IEEE Infocom 05, pp. ??, 2005.

[19] J.K. Lee, J. Hou, "Modeling Steady-State and Transient Behaviors of User Mobility: Formulation, Analysis, and Application", Proc. ACM MobiHoc 06, to appear, 2006.

[20] D. Lelescu, U. Kozat, R. Jain, M. Balakrishnan, "Model T++: An Empirical Joint Space-Time Registration Model", Proc. ACM MobiHoc 06, to appear, 2006

[21] M. McGuire, "Stationary Distributions of Random Walk Mobility Models for Wireless Ad Hoc Networks", Proc. ACM MobiHoc 05, pp. 90-98, 2005.

[22] M. Musolesi, S. Hailes, C. Mascolo, "An Ad Hoc Mobility Model Founded on Social Network Theory", Proc. ACM MSWiM 04, pp. 20-24, 2004.

[23] W. Navidi, T. Camp, "Stationary Distributions for the Random Waypoint Mobility Model", IEEE Transactions on Mobile Computing, Vol. 3, n. 1, pp. 99-108, 2004.

[24] G. Resta, P. Santi, "The QoS-RWP Mobility and User Behavior Model for Public Area Wireless Networks", Tech. Rep. IIT-TR-03/2006, Istituto di Informatica e Telematica, Pisa - Italy, May 2006.

[25] M. Satyanarayanan, "Pervasive Computing: Vision and Challanges", IEEE Personal Communications, Vol. 8, n. 4, pp. 10-17, August 2001.

[26] D. Tang, M. Baker, "Analysis of a Local-Area Wireless Network", Proc. ACM Mobicom, pp. 1-10, 2000.

[27] C. Tuduce, T. Gross, "A Mobility Model based on WLAN Traces and its Validation", Proc. IEEE Infocom 05, pp. 664-674, 2005.

[28] J. Yoon, M. Liu, and B. Noble, "Random Waypoint Considered Harmful", Proc. IEEE Infocom, (San Francisco, CA), April 2003.

[29] J. Yoon, M. Liu, and B. Noble, "Sound Mobility Models", Proc. ACM MobiCom, (San Diego, CA), pp. 205-216, Sept. 2003. 